\title{
Two kinds of two-dimensional order : the SmF and SmI phases (*)
}

\author{
J. J. Benattar, F. Moussa, M. Lambert (**) \\ Lab. Léon-Brillouin, CEN Saclay, 91191 Gif sur Yvette Cedex, France \\ and C. Germain
}

Lab. de Physique des Solides, Université Paris-Sud, Bât. 510, 91405 Orsay, France

(Reçu le 17 novembre 1980, accepté le 15 décembre 1980)

Résumé. - Une étude aux rayons X du TBDA, homologue du TBBA, montre que les phases smectiques $F$ et I présentent un ordre quasi-bidimensionnel (2D). L'analyse quantitative des spectres de poudre met en évidence dans la phase $\mathrm{SmF}$ un ordre à courte distance et dans la phase $\mathrm{SmI}$, quoique à plus haute température, un ordre à grande distance. Cette phase pourrait être un exemple de solide 2D.

Abstract. - A X-ray study of TBDA, a homologous compound of TBBA, shows that the smectic F and I phases exhibit a quasi-two-dimensional order (2D). A quantitative analysis of the powder diagrams yields short range order in the SmF phase and long range order in the SmI phase though at higher temperatures. This phase could be an example of a $2 \mathrm{D}$ solid.

In recent years, two-dimensional (2D) systems have been the subject of a growing interest. New « liquid crystal » phases, recently discovered, provide good examples of such systems.

The synthesis of TBBA homologous compounds with the general formula

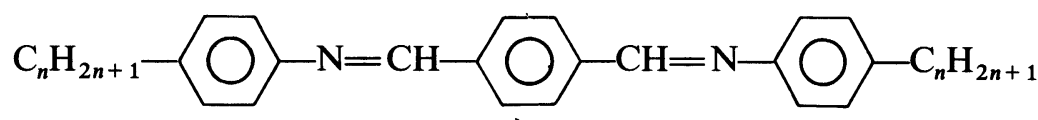

first allowed us to establish the existence of the smectic $\mathrm{F}$ phase $(\mathrm{SmF})$ in the $\mathrm{C}_{5}$ compound [1] then the existence of the smectic I phase (SmI) in the $\mathrm{C}_{9}$ and $\mathrm{C}_{10}$ compounds [2]. We have synthesized new homologous of the series according to the process used for TBBA [3].

These last compounds show the following polymorphism :

\begin{tabular}{|c|c|c|c|c|c|c|c|}
\hline $\mathrm{C}_{n} \quad \mathrm{Cr}_{1}$ & $\mathrm{Cr}_{2}$ & $\operatorname{SmG}(*)$ & $\mathrm{SmF}$ & SmI & $\mathrm{SmC}$ & $\operatorname{SmA}$ & $\mathrm{Li}$ \\
\hline $\begin{array}{l}C_{10} \\
C_{13}-50 \\
C_{15}-85\end{array}$ & $\begin{array}{r}-72 \\
C-85 \\
C-91\end{array}$ & $\begin{array}{l}C-112 \\
C-\sim 115 \\
C-\sim 117\end{array}$ & $\begin{array}{l}\mathrm{C}-148 \\
\mathrm{C}-\sim 130 \\
\mathrm{C} .\end{array}$ & $\begin{array}{r}C-156 \\
C-153 \\
-147\end{array}$ & $\begin{array}{l}C-186 \\
C-178 \\
C-170\end{array}$ & $\begin{array}{l}\mathrm{C}-189 \\
\mathrm{C} \\
\mathrm{C}\end{array}$ & $\begin{array}{l}\mathrm{C}- \\
- \\
-\end{array}$ \\
\hline
\end{tabular}

$\left(^{*}\right)$ We follow the advice of D. Demus et al. [13] concerning the labelling of the different smectic phases $\left(\mathrm{SmG} \equiv \mathrm{SmB}_{\mathrm{c}}\right)$.

The SmI phase appears between the SmF phase and the $\mathrm{SmA}$ or SmC phase, which consists of stacked

$\left(^{*}\right)$ Version anglaise d'une note présentée aux Comptes Rendus de l'Académie des Sciences le 17 novembre 1980.

$\left({ }^{* *}\right)$ Also : Laboratoire de Physique des Solides, Université Paris-Sud. layers of « liquid type ». (It should be noted that the $\mathrm{C}_{15}$ compound does not have a SmF phase.)

The purpose of this letter is to study the change in order at the $\mathrm{SmF} \leftrightarrow \mathrm{SmI}$ transition in the $\mathrm{C}_{10}$ compound (TBDA). An accurate quantitative analysis of the order cannot be done on single domains, because such samples often exhibit a large and unknown 
mosaic spread - this has been avoided by recording Debye-Scherrer diagrams on a high resolution Guinier camera $\left(\mathrm{Cok}_{\alpha_{1}}, \lambda=1.7889 \AA\right)$.

Moreover, we must point out that the differential thermal analysis has shown a great enthalpy variation at the $\mathrm{SmI} \leftrightarrow \mathrm{SmC}$ transition and a weak variation at the $\mathrm{SmF} \leftrightarrow \mathrm{SmI}$ transition, which means that the main change of order takes place at the transition : $\mathrm{SmI} \leftrightarrow \mathrm{SmC}$

1. Structures of the SmF and SmI phases. -

a) THE SmF PHASE. - X-ray patterns previously recorded $[4,5,6]$ enabled us to assert the following : i) the layered structure of this phase has a tilt angle of the molecules with respect to the layer normal; ii) there is a very weak coupling between adjacent layers (as a matter of fact there do not exist $h k l$ spots with $l \neq 0$ : so, the structure is two-dimensional kind); iii) locally there is pseudo-hexagonal packing of the molecules within the layers. (Indeed the $h k 0$ Bragg peaks which are on a nearly regular hexagon (distorded by one percent) in the SmG phase [7] become diffuse spots in the SmF phase, centred of the same points of the reciprocal space.)

Though the in-plane order does not extend to a long distance, and though the layers are very weakly coupled, one defines a local cell which is monoclinic with centred $\mathrm{C}$-faces and its corresponding reciprocal lattice (Fig. 1b).

The cell parameters of TBDA $\left(\mathrm{C}_{10}\right)$ at $T \cong 120^{\circ} \mathrm{C}$ are :

$$
\begin{array}{ll}
a=9.64 \pm 0.02 \AA, & b=5.22 \pm 0.01 \AA, \\
c=41.7 \pm 1 \AA, & \beta=112^{\circ} \pm 2^{\circ} .
\end{array}
$$
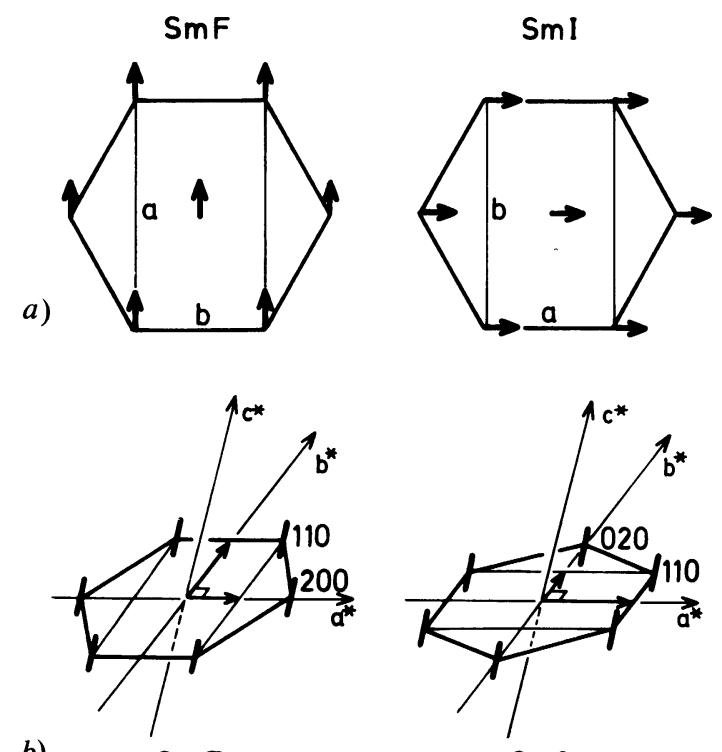

$\mathrm{SmF}$
In this phase the molecules are tilted along the a direction, $a$ being the largest side of the rectangular basis (Fig. 1a).

b) THE SmI PHASE. - Powder diagrams of the SmI phase at $T \cong 152^{\circ} \mathrm{C}$ are analogous, at first sight, to those of the $\mathrm{SmF}$ phase. One observes the very sharp 001 spots characteristic of a layered structure, and a ring at large scattering angles. On the other hand, the diffuse ring is sharper than the corresponding one in the SmF phase, so the order within the layers extends to a longer distance in the SmI phase than in the $\mathrm{SmF}$ phase, whereas the temperature range for the SmI phase is higher than for the $\mathrm{SmF}$ phase $\left({ }^{1}\right)$.

The X-ray diagrams given by single domain samples of this phase $[2,8]$ have shown that within the layers, the packing is still pseudo-hexagonal and one can define a local C-centred monoclinic cell which is very different from that of $\mathrm{SmF}$, since here the molecules are tilted toward the smaller side of the rectangular basis (Fig. 1a). Figure $1 b$ describes the reciprocal lattice related to this structure. The cell parameters are, at $T \simeq 152^{\circ} \mathrm{C}$ :

$$
\begin{aligned}
& a=5.62 \pm 0.01 \AA, \quad b=9 \pm 0.02 \AA, \\
& c=41 \pm 1 \AA, \quad \beta=111^{\circ} \pm 2^{\circ} .
\end{aligned}
$$

2. Method of analysing the line shape of the diffuse ring. - As already noted, the large angle ring is characteristic of the order of the molecules in the smectic layers. The structure of the SmF phase being only pseudo-hexagonal, the ring is therefore composed of the two 110 and 200 spots which have nearly the same modulus of the scattering wave vector, whereas in the SmI phase (the long axis of the molecules being along another direction) the ring results from the superposition of the 020 and 110 spots (Fig. 1b). Moreover, for a powder diagram, the multiplicity of the 110 spot is twice as large as the multiplicity of the 020 or 200 spots. The line shape of the ring is obtained by adding both spots which compose it, weighted by the multiplicity.

We have shown [5] that the intensity distribution $I_{\mathbf{G}}(Q)$ ( $\mathbf{Q}$ being the scattering vector) can be obtained, in the case of a 2D system, by summing the intensity located around $\mathbf{G}=h \mathbf{a}^{*}+k \mathbf{b}^{*}$ and extending along $\mathbf{c}^{*}$ (c* being parallel to the layer normal), over a sphere whose radius is $Q$ (Fig. 2) such as :

$$
I_{\mathbf{G}}(Q)=\int \mathrm{d}^{3} k S(\mathbf{k}) \delta\left(|\mathbf{k}|^{2}-|\mathbf{Q}|^{2}\right)
$$

where the scattering function $S(\mathbf{k})$ can be written as the product of the scattering function in the $\left(\mathbf{a}^{*}, \mathbf{b}^{*}\right)$ plane $f_{\mathbf{G}}\left(q_{x}, q_{z}\right)$ by the intensity distribution along $\mathrm{c}^{*}$.

In the $\mathrm{SmF}$ and SmI phases the layers are uncorrelated, and thus the intensity scattered by all the layers is proportional to the intensity scattered by one layer.

( $\left.{ }^{1}\right)$ This increase in the order at higher temperatures has been already observed in the reentrance phenomena [9].
Fig. 1. - a) Molecular packing and local cell within the layer plane of the SmF and SmI phases. The arrows represent the molecular projection on this plane. b) Reciprocal lattices associated to the figure $a$. The bars show the direction of the maximum extension of the diffuse spots. 


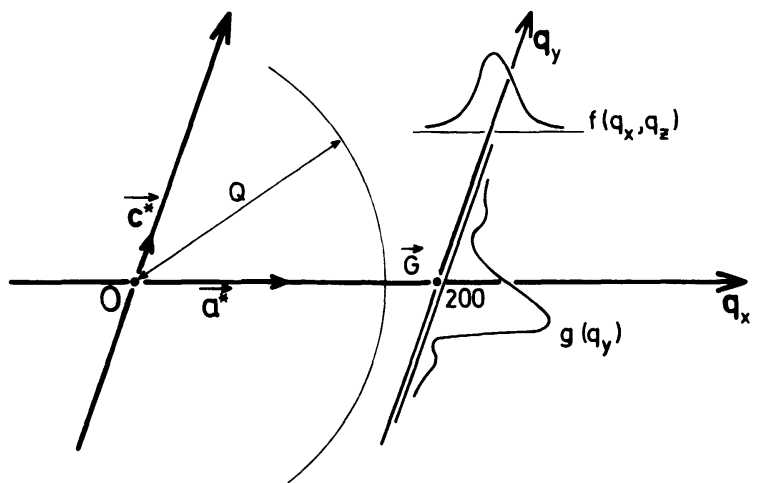

Fig. 2. - Intersection of a sphere (radius $Q$ ) with the $\left(\mathbf{a}^{*}, \mathbf{c}^{*}\right)$ plane. The scattered intensity is localized near the point $\mathbf{G}=(2,0,0)$, where $\mathbf{q}=\mathbf{Q}-\mathbf{G}$.

The intensity, in the $\mathbf{c}^{*}$ direction, is then given by the square of the Fourier transform of the molecular electronic density $\rho$. We describe $\rho$ as the sum of two step-functions characteristic of the electronic density of the core and of the alphatic chains [5].

3. Discussion of the results. - The theory of 2D melting built by Halperin and Nelson [10], upon ideas of Kosterlitz and Thouless [11], predicts two steps : above a first critical temperature, the loss of positional order occurs, above a second critical temperature, the loss of orientational order also occurs, the system becoming an isotropic 2D liquid.

It is well known that a true « $2 \mathrm{D}$ solid » can exist only at zero temperature. However, from a lowtemperature development, one can show that the pair positional correlation function follows a power law : $r^{-\eta_{G}(T)}$ at finite temperature, and the usual Bragg peaks are replaced by $|\mathbf{Q}-\mathbf{G}|^{-2+\eta_{G}(T)}$ [12] at a set of reciprocal lattice vectors $\mathbf{G}$; moreover, this system has a long range orientational order.

Above a first critical temperature, an unbinding of dislocation pairs occurs, giving rise to an exponential decay of the translational order $: \exp (-r / \xi)(\xi$ being the correlation length of the translational order in the layers). In this phase, called " hexatic " by Halperin and Nelson, the scattering function becomes $\propto\left[\xi^{2}|\mathbf{Q}-\mathbf{G}|^{2}+1\right]^{-1}$; furthermore, the orientational order follows an algebraic decay.

In the second step of the 2D melting, the unbinding of disclination pairs leads to an isotropic 2D liquid phase where both translational and orientational orders fall exponentially.

a) THE SmF PHASE. - In a previous paper on the TBPA SmF phase, it has been shown that the line shape of the diffuse ring can be analysed following the method described in paragraph 2, choosing a Lorentzian as the in-plane structure factor :

$$
f_{\mathbf{G}}\left(q_{x}, q_{z}\right) \propto\left[\xi^{2}\left(q_{x}^{2}+q_{z}^{2}\right)+1\right]^{-1} .
$$

One finds the same results on TBDA.
On figure 3, we compare the experimental intensity measured at $T=120 \pm 2{ }^{\circ} \mathrm{C}$ with the one calculated according to the formula (1), using a Lorentzian structure factor. For a correlation length

$$
\xi=250 \pm 30 \AA
$$

there is a good agreement.

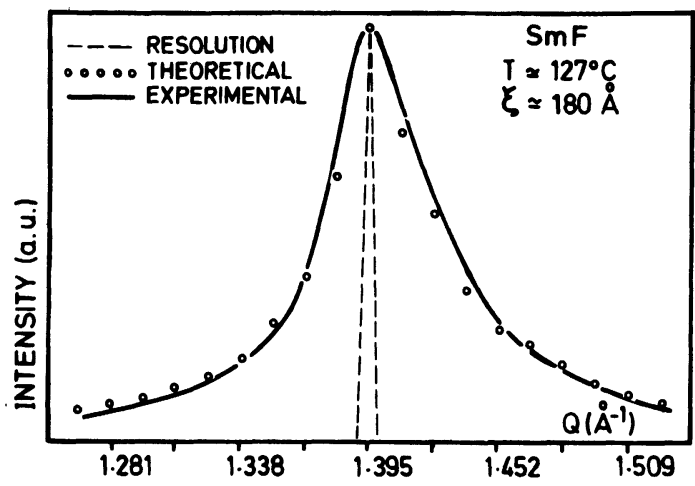

Fig. 3. - Comparison of the line shape measured in the SmF phase of TBDA with the calculated one, the in-plane scattering function being a Lorentzian.

Another fit of measurements at $T=127 \pm 2{ }^{\circ} \mathrm{C}$ gives : $\xi=180 \pm 20 \AA$. We must point out that, whatever the homologous compound, the correlation length characteristic of the short range order within the layers, has the same order of magnitude. We have also reported, on figure 3 , the apparatus function - its width is small if compared with that of the experimental line shape, and then it does not contribute to the broadening.

b) The SmI PHASE. - When one investigates the line shape of the diffuse ring in the SmI phase, one can see that the width at middle-height is weaker than the width measured in the SmF phase - the SmI phase therefore seems much well ordered than the $\mathrm{SmF}$ although it appears at higher temperature. A first attempt to fit the experimental data with a Lorentzian structure factor failed, whatever the value of the correlation length. So, we employed a power law decay

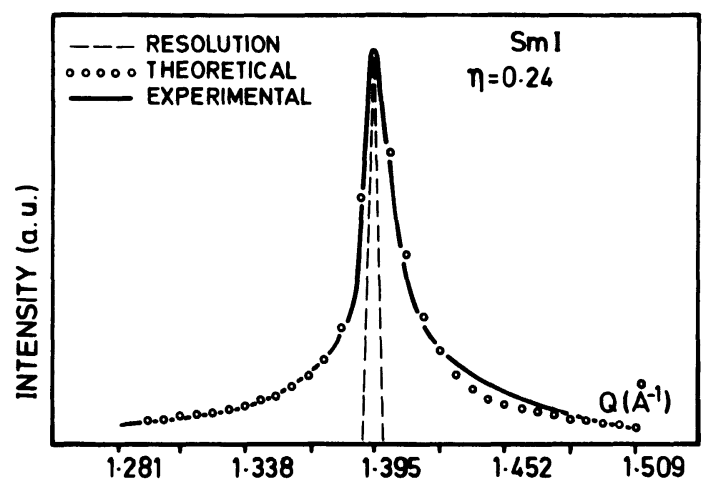

Fig. 4. - Comparison of the line shape measured in the SmI phase of TBDA with the calculated one, the in-plane scattering function being $\propto\left(q_{x}^{2}+q_{z}^{2}\right)^{(-2+\eta) / 2}$. 
$\left(q_{x}^{2}+q_{z}^{2}\right)^{(-2+\eta) / 2}$. As this law diverges, one must perform the convolution of the scattering function with the resolution function of the spectrometer. Except in a range of $\pm 0.01 \AA^{-1}$ around the $Q$ value corresponding to the maximum of the scattered intensity, the resolution function has no drastic effects. We have compared our calculations to the "wings » of the spectrum (Fig. 4). The value of $\eta$ which gives the best agreement is $\eta=0.24 \pm 0.02$ at $T=152^{\circ} \mathrm{C}$, which is comparable with the upper limit 0.25 found in the theory [11]. The temperature range of this phase being only $8^{\circ} \mathrm{C}$, the temperature dependence of $\eta$ is undetectable within the precision of the fit.

The $\mathrm{C}_{15}$ compound would allow a study of the temperature dependence of $\eta$ since the SmI temperature range is larger.

In conclusion, we have shown, for the first time, that the SmI phase of TBDA exhibits a « topological » long range order characteristic of a $2 \mathrm{D}$ solid, whereas in the $\mathrm{SmF}$ phase there is a short range order. We have confirmed these results in the $C_{13}$ and $C_{15}$ homologous compounds.

\section{References}

[1] Goodby, J. W., Gray, G. W., Mosley, A., Mol. Cryst. Liq. Cryst. Lett. 41 (1978) 183.

[2] Diele, S., Demus, D., SaCkmann, H., Mol. Cryst. Liq. Cryst. Lett. 56 (1980) 217.

[3] LIEBERT, L., Liq. Cryst. Solid State Phys. Supplement 14 (Academic Press, New York) 1978.

[4] Benattar, J. J., Doucet, J., Lambert, M. and Levelut, A. M., Phys. Rev. A 20 (1979) 2505.

[5] Benattar, J. J., Moussa, F. and Lambert, M., J. Physique 41 (1980) 1371.

[6] Leadbetter, A. J., Gaughan, J. P., Kelly, B., Gray, G. W., Goodby, J. W., J. Physique Colloq. 40 (1979) C3-178.

[7] Doucet, J., Levelut, A. M. and Lambert, M., Phys. Rev. Lett. 32 (1974) 301.

[8] Gane, P. A. C., Leadbetter, A. J. and Wrighton, P. G., to be published.
[9] Prost, J., Proceedings of the Conference on Liquid Crystal of one- and two-dimensional order and their applications, Garmisch-Partenkirchen, F. R. G. (Springer-Verlag) 1980, p. 125.

[10] Nelson, D. R. and Halperin, B. I., Phys. Rev. B 19 (1979) 2457.

[11] Kosterlitz, J. M. and Thouless, D. J., J. Physique Colloq. 34 (1973) C6-1181.

[12] De Gennes, P. G. and Sarma, G., Phys. Lett. A 38 (1972) 219.

[13] Demus, D., Goodby, J. W., Gray, G. W. and Sackmann, H., Proceedings of the Conference of Liquid Crystals of oneand two-dimensional order and their applications, Garmisch-Partenkirchen, F. R. G. (Springer-Verlag) 1980, p. 31. 